\title{
Mit dem Zwicker von Eugen Bleuler
}

Maike Rotzoll

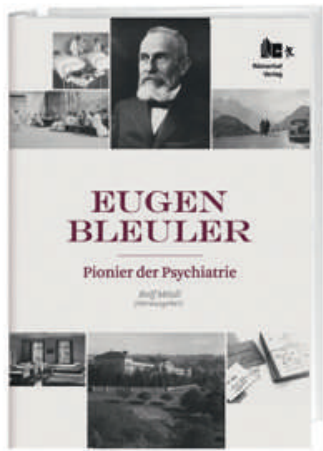

Rolf Mösli (Hrsg.) Eugen Bleuler -

Pionier der Psychiatrie

Zürich: Römerhof Verlag; 2012

206 Seiten, ca. 200 Abb., $44 \mathrm{CHF}$

ISBN 978-3-905894-12-7

Korrespondenz: Dr. med. Maike Rotzoll Institut für Geschichte und Ethik der Medizin RuprechtKarls-Universität Heidelberg Im Neuenheimer Feld 327 D-69120 Heidelberg

maike.rotzoll[at]histmed. uni-heidelberg.de
War das Altgriechische im 20. Jahrhundert eine «tote Sprache»? Ein ausserhalb der Welt angeblich verstaubter humanistischer Gymnasien fast vergessenes Idiom, jenseits von sprachlicher Kreativität? Von wegen!

Im Jahr 1908 prägte der Zürcher Psychiater Eugen Bleuler im Rückgriff auf die griechische Sprache den bald weltweit wirkmächtigen Begriff «Schizophrenie» («Spaltungsirresein») - und grenzte sich damit von seinem deutschen Kollegen Emil Kraepelin ab: Dieser hatte nur wenige Jahre zuvor für das gleiche Krankheitsbild den lateinischen Namen «Dementia praecoX» («vorzeitige Verblödung») gefunden. Dabei ging es um mehr als um Worte aus zwei unterschiedlichen antiken Sprachen. Die Prognose der vermuteten Krankheitseinheit Dementia praecox unerbittlich voraussehend, ordnete Kraepelin ein: Seine Systematik psychiatrischer Erkrankungen, am Klassifikationsschema von ihm hochgeschätzter Botaniker orientiert, prägte die Entwicklung des Faches weit über nationale Grenzen hinaus. Bleuler dagegen eliminierte den fatalen Ausdruck Demenz und bezog sich mit der Schizophrenie stärker auf das Erleben der Patientinnen und Patienten. So ermöglichte er nach den Worten seines Sohnes Manfred Bleuler den Betroffenen und ihren Angehörigen «Hoffnung auf Heilung».

Waren Emil Kraepelin und Eugen Bleuler wirklich so weit auseinander, was die Prognose dieser unterschiedlich benannten, besonders schwerwiegenden und häufigen psychischen Krankheit oder Gruppe von Krankheiten betraf? Der Heidelberger Psychiater Hans Walter Gruhle konstatierte 1913, Kraepelin habe eingeräumt, dass vollständige Heilungen vorkommen könnten, jedoch sehr selten seien. Bleuler dagegen halte eine Restitution auf den vor einem Schub herrschenden Zustand für nicht selten, eine vollständige Heilung habe er jedoch noch nie gesehen. Bleulers Krankheitsbegriff setzte sich jedenfalls rasch durch, doch galten Schizophrene noch lange weitestgehend als chronisch kranke, als unheilbare Langzeitpatientinnen und -patienten. Trifft das Attribut Pionier, im Titel des hier zu besprechenden Buches auf den Zürcher Psychiatrieprofessor angewandt, nicht viel eher deswegen zu, weil Bleuler die Universitätsklinik Burghölzli früh für psychoanalytisch ausgerichtete Ärzte und ihre Therapien öffnete?

Ganz sicher ist Bleuler eine so bedeutsame Gestalt der Psychiatriegeschichte, dass sich auch ein sehr persönlicher Blick auf seine Biographie und die Entwick-

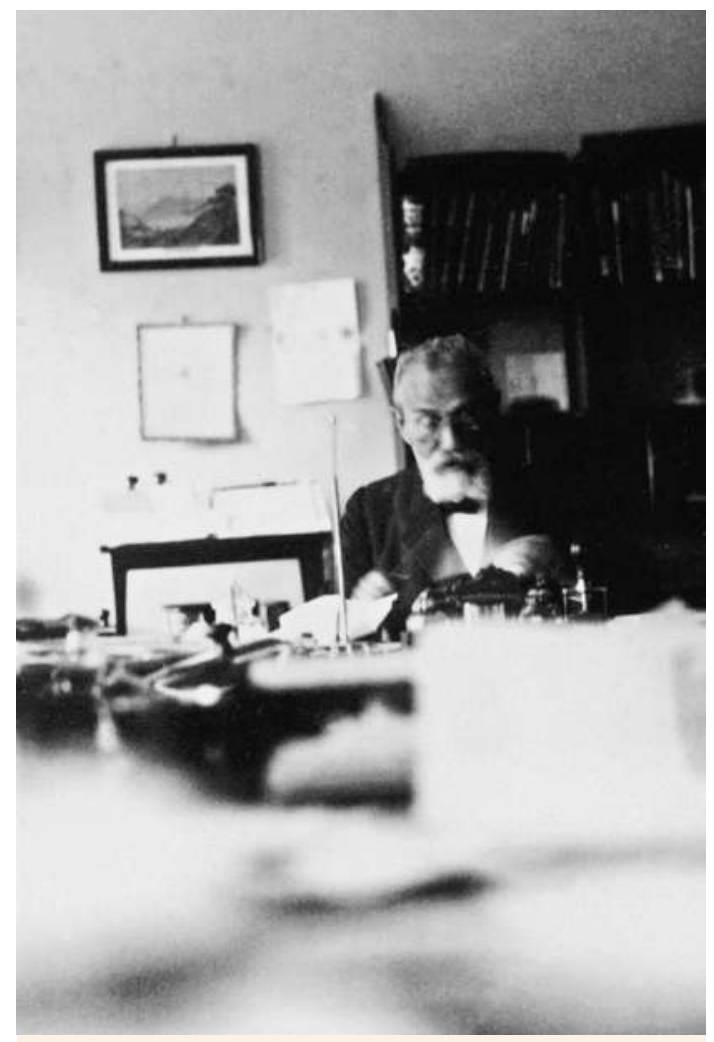

Bleuler in der Amtsstube (Privatalbum Familie Bleuler).

lung der von ihm geleiteten Institutionen Rheinau und Burghölzli lohnt, wie ihn sich Rolf Mösli, der Herausgeber des vorliegenden Buches, vorgenommen hat.

Nun ist es allerdings nicht so, dass Bleulers bedeutsamer Beitrag zur psychiatrischen Ideengeschichte oder die Geschichte psychiatrischer Institutionen im Kanton Zürich bislang keinen Widerhall in der Geschichtsschreibung gefunden hätten. 2007 erst hat eine Gruppe Zürcher Sozialhistoriker um Marietta Meier unter dem Titel «Zwang zur Ordnung. Psychiatrie im Kanton Zürich, 1870-1970» eine sehr differenzierte Studie über das Burghölzli und die Rheinau veröffentlicht, die weniger auf die beteiligten Ärzte als auf die Strukturen der Institutionen und ihre Auswirkungen auf die Patientinnen und Patienten fokussiert. Gleichwohl thematisiert die Studie Bleulers Beiträge zur Psychiatriegeschichte, stellt die «Erfolgsgeschichte» jedoch in einen weiteren und kritischen Kontext. Das problematische Doppelmandat der Psychiatrie, ihre Zuständigkeit für individuelles 
Leid ebenso wie für Ordnungsbedürfnisse der Gesellschaft, wird in diesem Buch programmatisch «in seiner Ambivalenz» dargestellt. Die Autorinnen und Autoren ergreifen nicht Partei für die optimistisch auf die Entwicklung der heilenden Psychiatrie blickende Fortschrittshypothese oder für die skeptisch auf zunehmende gesellschaftliche Kontrolle fokussierenden Deutungsmuster der Sozialdisziplinierung, sondern neuere Forschungsliteratur repräsentieren, zu finden. Man gerät ins Spekulieren, ob sie für Mösli zu der in seiner Einleitung andeutungsweise genannten Fachliteratur gehören könnten, in der man «vereinzelt gewagt spekulative [...] Analysen über Eugen Bleulers Psychopathologielehre und seine allgemeinen Ansichten» lesen könne. Von nicht genannten Autoren grenzt sich Mösli dadurch ab, dass er sich vornimmt,

\section{Man kann dieses Buch fast durchwandern wie ein kleines psychiatriehistorisches Museum.}

sie stellen historische und kulturelle Kontexte her. Dabei werden auch eugenische Debatten zu Eugen Bleulers Zeit («soziale Sterilisation») und die Sterilisationspraxis am Burghölzli in den 1930er Jahren oder die Psychochirurgie der Zeit nach dem Zweiten Weltkrieg nicht ausgespart, ebenso wenig wie die Sicht der Patientinnen und Patienten auf ärztliche Massnahmen und Anstaltsordnung [1].

Patientengeschichtlich orientiert ist auch ein weiteres Werk: Im Jahr 2010 dokumentierte die Ausstellung «Rosenstrumpf und dornencknie. Werke aus der Psychiatrischen Pflegeanstalt Rheinau 1867-1930» ebenso wie der von der Kunsthistorikerin Karin Luchsinger et al. herausgegebene Ausstellungskatalog mit Werken von Patienten-Künstlern auch einen Ausschnitt der Patientensicht auf das Leben in der Pflegeanstalt auf der Rheininsel [2].

Es erstaunt, in Möslis Buch keinen Verweis auf diese beiden thematisch einschlägigen Werke, die

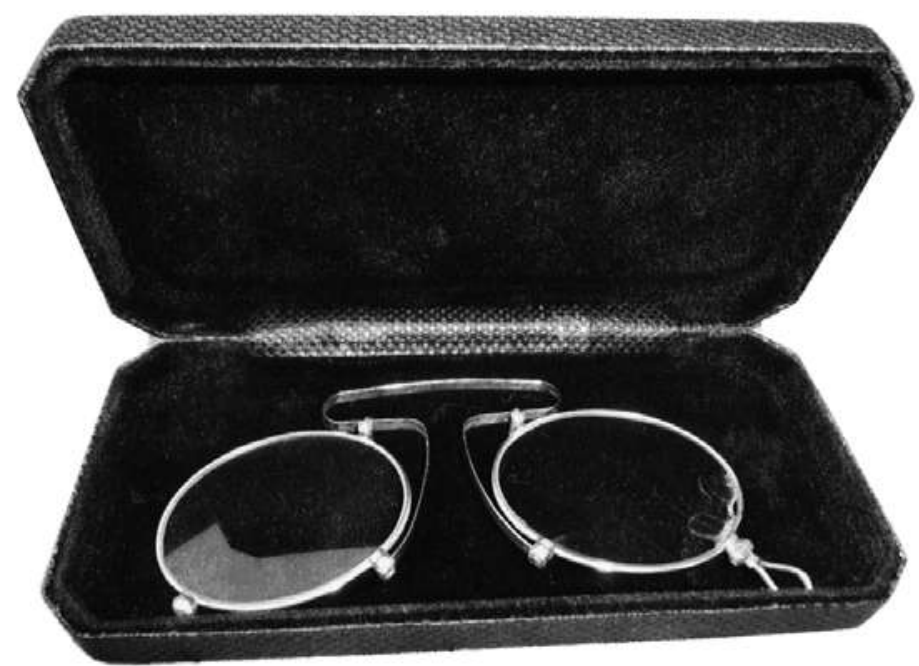

Der Zwicker von Eugen Bleuler (Privatarchiv Rolf Mösli). «die Fachwelt und alle an Psychiatrie Interessierten für einmal auch mit der menschlich-emotionalen Seite dieses grossen Mannes» vertraut zu machen.

«Beiträge zu Eugen Bleulers Wirken und Denken gab es schon früher», so formuliert Daniel Hell, einer von Bleulers Nachfolgern, in seiner Einleitung. Doch es fehlten nach seiner Ansicht bislang Bildmaterialien und weitere Angaben zur Familien- und persönlichen Geschichte Eugen Bleulers. Diese Lücke schliesse der Band, der sich hauptsächlich der jahrzehntelangen Sammeltätigkeit Rolf Möslis verdanke. Mösli sei ebenso die dankenswerte Initiative für das psychiatriehistorische Museum im Burghölzli zuzuschreiben. In der Tat erschliesst sich das Buch vor allem über die Fotografien. In ihrer Dichte und Intensität scheinen sie die Welt von damals so plastisch werden zu lassen, dass man fast meint, man sähe sie mit dem Zwicker von Eugen Bleuler, der bis heute erhalten geblieben ist. Auch der Zwicker wird, gleich einer Reliquie, im Buch liebevoll mit einem Foto dokumentiert.

Eine wahre Fundgrube von visuellen Eindrücken also ist Möslis Buch in jedem Fall. Die Fotos dokumentieren dabei nicht ausschliesslich Bleulers Familiengeschichte sowie Personen aus seinem beruflichen Umfeld, sondern durchaus auch Situationen aus dem psychiatrischen Alltag. Hierbei bezieht sich Mösli, selbst ehemaliger Psychiatriepfleger, besonders auch auf die Perspektive der nicht-ärztlichen Mitarbeiter und Mitarbeiterinnen. Diese Gruppe wird erfreulicherweise auch in jenen Texten besonders berücksichtigt, die Mösli selbst verfasst hat. Dabei wird deutlich, dass er als Zeitzeuge schreibt, aus einer Binnenperspektive jedenfalls. Zwar hat er Eugen Bleulers Zeit nicht selbst miterlebt, jedoch hat er offenbar den Geist des Hauses verinnerlicht. Fast jeder Satz verrät seine Identifikation mit Bleuler und der von ihm geleiteten Institution.

Das Buch vereinigt sehr unterschiedliche Texte. Nach einem ausführlichen und gut lesbaren Beitrag von Daniel Hell zu Bleulers familiärer Herkunft aus bäuerlichem Milieu, seiner Kindheit und Jugend informiert Mösli sehr knapp auf einer Doppelseite über 


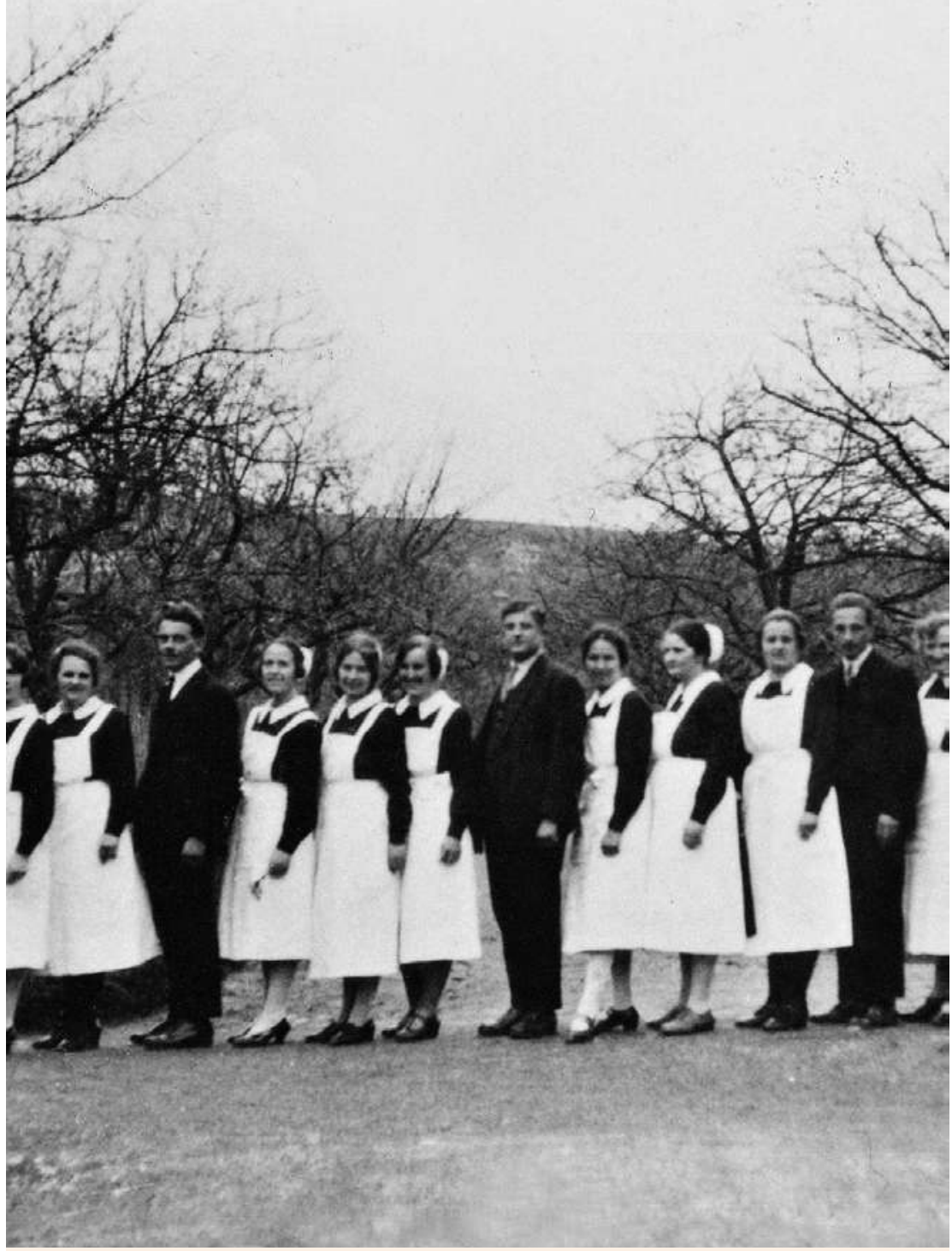

Schwestern des Burghölzli in Sonntagstracht (Bildarchiv Burghölzli).

die Geschichte der Pflegeanstalt Rheinau, Bleulers erster beruflicher Station. Dann kommt der «Pionier» selbst zu Wort: Vier Texte Bleulers werden, farblich zartrosa abgesetzt, erneut abgedruckt, unterbrochen nur von Gottfried Kellers kurzem Prosatext «Die Weihnachtsfeier im Irrenhaus 1879», deren Handlung im Burghölzli spielt. Zwei der Bleuler-Texte sind von psychiatriehistorischem Interesse: «Die Erweiterung der zürcherischen Irrenpflege» und «Die allgemeine Behandlung der Geisteskranken». Die beiden anderen - «Ethik Glauben Wissen» und «Das Leben nach dem Tode» behandeln allgemeinere Lebensfragen auf für Bleuler charakteristische Art.

Bei Rolf Möslis Kapitel «Die bewegende Geschichte des Burghölzli» handelt es sich um eine detailreiche Zeittafel. Das anschliessende Kapitel, ebenfalls von Mösli, trägt den Titel «Die «Bleuler-Anstalt»». Hier berichtet Mösli zunächst von eigener
Berufserfahrung, um dann die historische Entwicklung des Wärterberufs zu skizzieren. Grössere Teile des Kapitels bestehen aus langen und eindrucksvollen Zitaten aus Josef Henggelers Bericht «Über die Stellung des Irrenpflegers im Anstaltsbetrieb» von 1924. Es folgen, wiederum farblich abgesetzt, alltagsgeschichtlich interessante Auszüge aus den Anfang der 1930er Jahre entstandenen Erinnerungen der ehemaligen Oberköchin des Burghölzli, Elisabeth Duttweiler-Gehrig.

Eugen Bleulers Schizophrenielehre würdigt schliesslich der Zürcher Psychiater und Philosoph Paul Hoff ausführlich in einer anschaulichen und allgemeinverständlichen Weise. Dieser informative und gut lesbare Aufsatz endet mit einem Resümee, in dem Bleuler reflektiert und nachvollziehbar ein nach wie vor bedeutsamer Platz in der Psychiatriegeschichte zugewiesen wird. Den Abschluss des Buches bilden vorwiegend private Erinnerungen mehrerer Familienmitglieder und Informationen über Eugen Bleulers Nachkommen. Ein knappes Literaturverzeichnis, ein Bildnachweis, ein Personenregister und ein Stammbaum runden das Buch ab.

Insgesamt richtete sich Mösli zwar mit seinem Buch, wie er in der Einleitung festhielt, an die Fachwelt ebenso wie an ein Laienpublikum. Doch nicht nur der Verzicht auf einen Anmerkungsapparat zeigt, dass kaum ein wissenschaftlicher Anspruch erhoben werden soll. Vor allem ist es die Konzentration auf die Binnenperspektive in Verbindung mit einer fortschrittsoptimistischen Grundhaltung, die deutlich machen: Hier soll ein breites Publikum angesprochen werden, implizit wohl unter anderem mit dem Ziel, durch ein positives Bild von der Entwicklung der Psychiatrie zu ihrer Entstigmatisierung beizutragen leider nach wie vor ein ebenso notwendiges wie wichtiges Anliegen.

Man kann dieses Buch fast durchwandern wie ein kleines psychiatriehistorisches Museum, eine Wunderkammer, in der nicht ohne Stolz die Errungenschaften des eigenen Hauses präsentiert werden und dies wiederum dürfte auch für (Psychiatrie-)Historiker nicht ohne Interesse sein. Einen bleibenden visuellen Eindruck erzeugen vor allem die vielfältigen Fotografien und Dokumente, nur einige von ihnen sind etwas zu klein abgedruckt. Besser könnte man sie lesen und verstehen, hätte man ihn zur Hand: den Zwicker von Eugen Bleuler.

\section{Literatur}

1 Meier M, Bernet B, Dubach R, Germann U. Zwang zur Ordnung. Psychiatrie im Kanton Zürich, 1870-1970. Zürich: Chronos; 2007.

2 Luchsinger K, Blum I, Fahrni J, Jagfeld M (Hrsg.). Rosenstrumpf und dornencknie. Werke aus der Psychiatrischen Pflegeanstalt Rheinau 1867-1930. Zürich: Chronos; 2010. Vgl. auch Rotzoll M. «Rosenstrumpf und dornencknie. Schweiz Ärztezeitung. 2011; 92(25):971-3. 